\title{
Women with type 2 diabetes mellitus have lower cortical porosity of the proximal femoral shaft using low-resolution CT than nondiabetic women, and increasing glucose is associated with reduced cortical porosity
}

\author{
Marit Osima ${ }^{\mathrm{a}, \mathrm{b}}$ MD \\ Rita $\mathrm{Kral}^{\mathrm{c}} \mathrm{MD}$ \\ Tove T Borgen ${ }^{\mathrm{d}} \mathrm{MD}$ \\ Ingvild K Høgest $\varnothing 1^{\text {e,f }}$ MD \\ Ragnar M Joakimsen ${ }^{\mathrm{g}, \mathrm{h}} \mathrm{MD}, \mathrm{PhD}$ \\ Erik F Eriksen ${ }^{\mathrm{e}, \mathrm{f}} \mathrm{MD}, \mathrm{PhD}$ \\ Åshild Bjørnerem ${ }^{\mathrm{c}, \mathrm{g}} \mathrm{MD}, \mathrm{PhD}$
}

Short title: Type 2 diabetes mellitus and cortical porosity

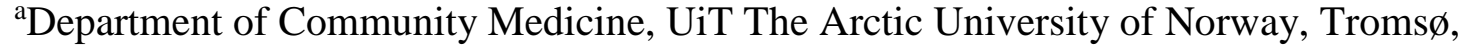
Norway

${ }^{\mathrm{b}}$ Department of Orthopaedic Surgery, University Hospital of North Norway, Troms $\varnothing$, Norway ${ }^{c}$ Department of Obstetrics and Gynaecology, University Hospital of North Norway, Troms $\varnothing$, Norway

${ }^{\mathrm{d} D e p a r t m e n t ~ o f ~ R h e u m a t o l o g y, ~ V e s t r e ~ V i k e n ~ H o s p i t a l ~ T r u s t, ~ H o s p i t a l ~ o f ~ D r a m m e n, ~}$ Drammen, Norway

${ }^{\mathrm{e}}$ Department of Endocrinology, Morbid Obesity and Preventive Medicine, Oslo University Hospital, Oslo, Norway

${ }^{\mathrm{f}}$ Department of Clinical Medicine, University of Oslo, Oslo, Norway

${ }^{g}$ Department of Clinical Medicine, UiT The Arctic University of Norway, Troms $\emptyset$, Norway

${ }^{\mathrm{h}}$ Department of Medicine, University Hospital of North Norway, Troms $\varnothing$, Norway
\end{abstract}

The North Norwegian Health Authorities funded the study (ID 5645, ID 9167, ID 9168, ID 10295) but had no role in the design and conduct of the study; in the collection, analyses, and interpretation of the data; or in the preparation, review, or approval of the manuscript.

Corresponding author:

Marit Osima

Department of Community Medicine, Faculty of Health Sciences,

UiT, The Arctic University of Norway, N-9037 Troms $\varnothing$, Norway

Tel +4799711178

Email:mos015@uit.no 


\section{Abstract}

Increased cortical porosity has been suggested as a possible factor increasing fracture propensity in patients with type 2 diabetes mellitus (T2DM). This is a paradox because cortical porosity is generally associated with high bone turnover, while bone turnover is reduced in patients with T2DM. We therefore wanted to test the hypothesis that women with T2DM have lower bone turnover markers (BTM) and lower cortical porosity than those without diabetes, and that higher serum glucose and body mass index (BMI) are associated with lower BTM, and with lower cortical porosity.

This cross-sectional study is based on a prior nested case-control study including 443 postmenopausal women aged 54-94 years from the Troms $\varnothing$ Study, 211 with non-vertebral fracture and 232 fracture-free controls. Of those 443 participants, 22 women exhibited T2DM and 421 women did not have diabetes. All had fasting blood samples assayed for procollagen type I N-terminal propeptide (PINP), C-terminal cross-linking telopeptide of type I collagen (CTX) and glucose, and femoral subtrochanteric architecture was quantified using lowresolution clinical CT and StrAx1.0 software.

Women with T2DM had higher serum glucose (7.2 vs. $5.3 \mathrm{mmol} / \mathrm{L}), \mathrm{BMI}\left(29.0 \mathrm{vs} .26 .4 \mathrm{~kg} / \mathrm{m}^{2}\right)$, and higher femoral subtrochanteric total volumetric bone mineral density (vBMD) (783 vs. 715 $\mathrm{mg} \mathrm{HA} / \mathrm{cm}^{3}$ ), but lower cortical porosity (40.9 vs. $\left.42.8 \%\right)$ than nondiabetic women (all p < 0.05). Each standard deviation (SD) increment in glucose was associated with 0.10-0.12 SD lower PINP and CTX, and 0.13 SD lower cortical porosity (all $p<0.05$ ). Each SD increment in BMI was associated with 0.10-0.18 SD lower serum PINP and CTX, and 0.19 SD thicker cortices (all $\mathrm{p}<0.05)$.

Increasing glucose and BMI were associated with lower bone turnover suggesting that reduced intracortical and endocortical remodeling leads to reduced porosity and thicker cortices. Using low-resolution clinical CT, cortical porosity was lower in women with T2DM compared to 
women without diabetes. This indicates that other changes in bone qualities, not increased cortical porosity, are likely to explain the increased fracture propensity in patients with T2DM.

\section{Key Words}

Bone turnover markers, cortical porosity, glucose, postmenopausal women, type 2 diabetes mellitus 


\section{Introduction}

Type 2 diabetes mellitus (T2DM) and bone fragility are public health problems coexisting with increasing age $[1,2]$. The prevalence of both conditions have increased over the last years, and thus the burden on society $[3,4]$. Patients with T2DM tend to have higher body mass index (BMI) and areal bone mineral density (aBMD), and would therefore be expected to exhibit reduced risk of fracture. Despite normal or increased aBMD they show increased risk of any fragility fracture [5-8]. T2DM itself is a modest risk factor for fracture, however, given the large number of individuals with this disease, fracture remains a major clinical concern [9].

In T2DM patients, fracture risk is higher for a given level of BMD compared to individuals without diabetes [8]. The reasons for the increased risk for fracture in T2DM patients are not clear $[6,10]$. Increased cortical porosity of distal radius or distal tibia has been invoked as one possible factor [11-13]. However, it is hard to explain how individuals with T2DM can exhibit high porosity [14-17], since increased cortical porosity reflects increased bone turnover from intracortical surfaces lining the Haversian canals and the endocortical surfaces adjacent to the marrow cavity $[18,19]$. As T2DM is a condition with low bone turnover [20,21], patients with T2DM would rather be expected to exhibit reduced cortical porosity. In contrast, prior studies reported increased porosity in T2DM patients. However, the absolute differences in cortical porosity were small $(0.8-2.4 \%)$, they included small sample sizes and measured porosity at peripheral sites using high-resolution peripheral quantitative computed tomography (HRpQCT) [11-13].

As cortical porosity at peripheral sites may not be representative of central sites, we examined cortical porosity at the proximal femur, a common site of the most serious fragility fracture [22]. Studies using HR-pQCT to quantify porosity present low values of porosity ranging from 
$1 \%$ to $15 \%$ because of quantifying only porosity of the compact cortex and only pores over $100 \mu \mathrm{m}[11-13,16]$, although $60 \%$ of cortical pores are under $100 \mu \mathrm{m}$ in diameter [6,23-25]. This threshold-based image analysis underestimates porosity by including only empty voxels [24]. StrAx1.0 software quantifies porosity as a fraction of void regardless of size of the pores, and captures indirectly porosity produced by pores larger and smaller than $100 \mu \mathrm{m}$ in diameter. It accounts for partial volume effect by including not only void within total empty voxels, but also partly empty voxels [24]. By using StrAx1.0 software we quantify porosity not only of the compact cortex but also the transitional zone. It is thus more inclusive than the traditional HR-pQCT measurements and the porosity is higher than reported using other methods [24,26].

Common characteristics of patients with T2DM are hyperglycemia and obesity. Increased BMI is associated with reduced bone turnover markers (BTM) and increased aBMD $[17,18]$. Similar observations with glucose-loading have been made in healthy subjects [15]. However, it remains to be determined whether the serum levels of glucose, BMI, or both, influences cortical porosity, cortical thickness or other bone features. We therefore wanted to test the hypothesis that 1) postmenopausal women with $\mathrm{T} 2 \mathrm{DM}$ have lower bone turnover markers and lower cortical porosity than those without diabetes, and that 2) higher serum levels of glucose and BMI are associated with lower bone turnover markers, and with lower cortical porosity.

\section{Materials and methods}

\section{Study population}

The Troms $\varnothing$ Study is a single-center population-based study in Northern Norway, which conducted six surveys from 1974 to 2008 [27]. In 1994-95 (Tromsø 4), all 37,558 eligible inhabitants in Troms $\emptyset$ over 24 years of age were invited, and 27,158 subjects (72\%) 
participated. In the Troms $\emptyset 4$ participants, all non-vertebral fractures were registered from the X-ray archives of the University Hospital of North Norway between 1 January 1994 and 1 January 2010 [28]. In 2011, we designed a nested case-control study that included 264 postmenopausal women from the fracture registry, who had suffered at least one fracture of the hip, wrist, or proximal humerus after the age of 50, and 260 age-matched fracture-free controls selected randomly from the same Troms $\varnothing 4$ cohort, previously described in detail $[18,22,29]$. Women who were premenopausal, received bisphosphonates for osteoporosis, had hip prostheses, metal screws, or pathological fractures were excluded. Of those 524 participants, we further excluded 15 currently receiving hormone replacement therapy and 66 with movement artifacts during CT scanning. The current study is a cross-sectional study based on the nested case-controls study described above, that included 443 participants (211 fracture cases [4 hip, 181 wrist, and 26 proximal humerus] and 232 fracture-free controls), of those 22 had T2DM and 421 did not have diabetes. The median time since their fracture was 6.6 years (range 1-25). All participants provided written informed consent and all measurements were performed between November 2011 through January 2013. The study was approved by the Regional Committee of Research Ethics and was conducted in accordance with the World Medical Association Declaration of Helsinki.

\section{Variables}

A questionnaire included information concerning fractures after the age of 50 years, number of falls in the last 12 months, diseases, use of medication and lifestyle-factors such as exercise and smoking. The self-reported diagnosis and duration of T2DM was confirmed based on information in their medical records, and diabetic complications were also identified. None of the participants reported that they had type 1 diabetes mellitus. Height and weight were 
measured in light clothing without shoes, and BMI was calculated as weight/height ${ }^{2}$. Total hip and femoral neck (FN) aBMD was measured at the non-dominant side using DXA (GE Lunar Prodigy, Lunar Corporation, Madison, WI, USA), and coefficients of variation (CV) were 1.2\% and $1.7 \%$, respectively [22].

Fasting blood samples were collected between 8 and 10 am and assayed for serum glucose using Roche Diagnostics, Germany, with CV 0.5-1.6\%, insulin using Elecsys 2010 Modular Analytics E170, Roche Diagnostics, Germany, with CV 0.8-4.6\%, 25-hydroxyvitamin D (25(OH)D) using mass spectrometry, parathyroid hormone (PTH) using Immulite 2000, and procollagen type I N-terminal propeptide (PINP) and C-terminal cross-linking telopeptide of type I collagen (CTX) using electrochemiluminescence immunoassay, Elecsys 1010 Analytics, Roche Diagnostics, Germany, with CV of 3-8\%. Homeostatic model assessment of Insulin Resistance (HOMA-IR) was calculated using the following formula: (glucose multiplied by insulin) divided by 135 [30], and kidney function was assessed using estimated glomerular filtration rate (eGFR).

CT scans (Siemens Somatom Sensation 16, Erlangen, Germany) of the non-dominant hip were performed at the Department of Radiology, University Hospital of North Norway. The CT machine had an in-plane resolution of $0.74 \mathrm{~mm}$, the slice thickness was $0.6 \mathrm{~mm}$, the hip was scanned from just above the femoral head to $2 \mathrm{~cm}$ below the lesser trochanter and the exposure dose of radiation was $\sim 1.5 \mathrm{mSv}$ [22]. CT scans of the hip were performed at $120 \mathrm{kV}$, a pitch of 0.75, using $90 \mathrm{~mA}$, reconstructed using a fixed field of view at $120 \mathrm{~mm}$ [31]. Quality control was carried out by scanning a phantom containing rods of HA (QRM Quality Assurance in Radiology and Medicine GmbH, Moehrendorf, Germany). These low-resolution CT images were analyzed in Melbourne (Australia) using StrAx1.0 software (StraxCorp Pty Ltd, 
Melbourne, Australia), a non-thresholding method, which automatically selects attenuation profile curves and segments the bone into compact-appearing cortex, inner and outer transitional zones (TZ), and trabecular compartment (Fig. 1) [32]. This is performed similarly in low-resolution images [22,26,32] as in HR-pQCT images [24,33]. As cortices are thin at the most proximal femur (femoral head, neck and trochanter), analyses were confined to the region of interest (ROI) where the cortices are thicker. This $3.7 \mathrm{~mm}$ subtrochanteric region was standardized by starting at tip of lesser trochanter, and the ROI was segmented into its compartments [22,32]. Porosity within each cortical compartment was quantified automatically throughout the ROI using StrAx1.0 software [22,24-26,29,31-33]. StraxCorp was blinded to T2DM and fracture status at the time of the analysis with StrAx 1.0. Cortical compartments at the subtrochanteric site are shown in Fig. 1 and prior articles [22,32]. Local bone edges are identified as the beginning and the end of the rising and falling S-shaped portions of the curve enabling the delineation of the compartments [24]. Analyzing 3600 consecutive overlapping profiles around the perimeter of each cross-sectional slice, segments the compartments. The density profile curve produced has two plateaus; one corresponding to the compact-appearing cortex and one corresponding to the trabecular compartment. Between these plateaus is a descending S shaped curve or transition between the two plateaus. This is the transitional zone. The density profile curve is expressing the mineralized bone area as the percentage of total area within each column.

Porosity presented here is defined as and determined as the average void volume fraction within the total cortex (compact appearing cortex, outer and inner transitional zone (TZ)). The porosity quantified by this algorithm is the proportion of emptiness within each voxel or the fraction of the bone occupied by void (porosity) [22,24,32]. StrAx 1.0 quantifies porosity in low-resolution CT even though pores are not visible to the naked eye and it is thus an indirect measure of 
porosity $[18,22,26,29,32]$. The size and number of pores were not determined by using this software. Accuracy of porosity measurements at distal radius and distal tibia using HR-pQCT images with a voxel size of $82 \mu \mathrm{m}$ was validated against $\mu \mathrm{CT}$ images of cadaver specimens with a voxel size of $19 \mu \mathrm{m}$ as the gold standard [24]. Zebaze et al. also assessed accuracy of porosity quantified at the proximal femur in HR-pQCT images, against scanning electron microscopy (SEM) images of specimens collected at $2.5 \mu \mathrm{m}$ resolution as the gold standard [24]. The agreement $\left(\mathrm{R}^{2}\right)$ between HR-pQCT and these gold standards for quantification of porosity ranged from 0.87 to 0.99 . The in vivo precision of StrAx1.0 analysis of HR-pQCT images was tested by rescanning seven women four times [24]. The in vivo and ex vivo precision error was $<4.0 \%[24]$.

Accuracy of porosity measurements using clinical CT images with a voxel size of $740 \mu \mathrm{m}$ was validated by testing agreement with HR-pQCT measurements with a voxel size of $82 \mu \mathrm{m}$ as the gold standard $[22,32]$. The agreement $\left(\mathrm{R}^{2}\right)$ between $\mathrm{CT}$ and HR-pQCT ranged from 0.86 to 0.96 for quantification of porosity at the same femoral subtrochanteric site (range 40-95\%) [22,32]. $\mathrm{CV}$ for porosity of each cortical compartment were below $4.0 \%$. For ethical reasons, it was not possible to perform in vivo validation with rescanning of women on the same day. Therefore, we performed an additional validation by repositioning and rescanning a human hip phantom (consisting of a human pelvic skeleton embedded in plastic material) 10 times, with CV for the CT subtrochanteric bone parameters between 0.3 and 2.3\% [22]. Thus, StrAx 1.0 software provides accurate and reliable measurements of cortical porosity and other bone traits.

\section{Statistical analyses}

In this cross-sectional study, results are presented stratified by T2DM-status and by fracture status. All normally distributed continuous variables are presented as mean \pm standard deviation 
(SD). Remaining variables; trabecular BV/TV, serum insulin and HOMA-IR, are presented as median (range). In order to correct for skewed distribution, we used log-transformed trabecular BV/TV in the analysis. Differences between women with and without T2DM were assessed using analysis of covariance (ANCOVA), adjusted for age and fracture status. In sub-analysis, we compared diabetic women with and without fracture, and nondiabetic women with and without fracture using ANOVA, adjusted for age and BMI. Scatterplots of PINP, CTX, cortical porosity and cortical thickness as a function of serum glucose and BMI are presented. Linear regression analysis was used for associations of BTM and bone architecture (y), as a function of glucose and BMI (x) adjusted for age and fracture status. Standardized regression coefficients (STB) were used to facilitate the comparison of the strength of the associations between the exposure and endpoints. Analyses were performed using STATA Software package, v14 (StataCorp, LP, Texas, USA) and SAS software package, v9.3 (SAS Institute Inc., Cary, NC, USA). All tests were two-sided and $\mathrm{p}<0.05$ considered significant.

\section{Results}

\section{Women with type 2 diabetes mellitus compared with those without diabetes}

Women with T2DM exhibited higher fasting serum levels of glucose (7.2 vs. $5.3 \mathrm{mmol} / \mathrm{L}$ ) and insulin (102 vs. 55 pmol/L), higher HOMA-IR (5.3 vs. 2.2) and BMI (29.0 vs. $26.4 \mathrm{~kg} / \mathrm{m}^{2}$ ), and lower serum vitamin D (67.6 vs. $80.4 \mathrm{nmol} / \mathrm{L})$ than those without diabetes (all $\mathrm{p}<0.05$, Table 1). Moreover, they had higher total vBMD (783 vs. $\left.715 \mathrm{mg} \mathrm{HA} / \mathrm{cm}^{3}\right)$ and cortical vBMD (1073 vs. $1041 \mathrm{mg} \mathrm{HA} / \mathrm{cm}^{3}$ ), lower total cortical porosity (40.9 vs. $42.8 \%$ ) of the femoral subtrochanteric region (all $\mathrm{p}<0.05$ ). Serum levels of PINP in women with T2DM tended to be lower than in women without diabetes ( 40.4 vs. $46.8 \mathrm{ng} / \mathrm{mL}$ ), though this did not reach significance $(\mathrm{p}=0.100)$. All results were adjusted for age and fracture status. 


\section{Glucose, insulin, BMI, and bone turnover markers}

Each SD increment in serum glucose was associated with 0.12 and 0.10 SD lower serum PINP and CTX, respectively (both $\mathrm{p}<0.05$, Table 2, Fig. 2a). Each SD increment in serum insulin and HOMA-IR were each associated with 0.10 SD lower CTX $(\mathrm{p}<0.05)$, but not associated with PINP. Each SD increment in BMI was associated with 0.10 and 0.18 SD lower serum PINP and CTX, respectively (both $\mathrm{p}<0.05$, Table 2, Fig. 2b).

\section{Glucose, insulin, BMI, cortical porosity, and cortical thickness}

Each SD increment in serum glucose, insulin, HOMA-IR and BMI was associated with 0.120.19 SD higher total vBMD (all $\mathrm{p}<0.05$ ). Each SD increment in serum glucose was associated with 0.13 SD lower cortical porosity $(\mathrm{p}=0.006)$, while serum insulin and HOMA-IR were not associated with cortical thickness or porosity. Each SD increment in BMI was associated with 0.19 SD thicker cortices $(\mathrm{p}<0.001)$. All results were adjusted for age and fracture status. Excluding those with the highest serum glucose above $7 \mathrm{mmol} / \mathrm{L}$ or those above $10 \mathrm{mmol} / \mathrm{L}$ did not change the results, as glucose remained associated with cortical porosity $(\mathrm{p}<0.05)$.

The median duration of T2DM was 6.7 years (range 1.8-17.9), and duration of diabetes was inversely but non-significantly associated with cortical porosity $(\mathrm{STB}=-0.28, \mathrm{p}=0.222$, Fig. 3). Of 22 women with T2DM, seven were not on medication for diabetes, 12 used only oral antidiabetics, while three used both oral antidiabetics and insulin. Only three women with T2DM had complications (one neuropathy, two nephropathy), however these three patients had cortical porosity ranging from 38 to $43 \%$, which was close to the average levels of cortical porosity, and they had additionally comorbidity as cancer and hypertension. 
Women with T2DM had lower cortical porosity than those without diabetes $(p=0.033)$, and women with fracture had higher porosity than fracture-free controls $(\mathrm{p}<0.001$, Fig. 4). We stratified the analysis. In women with T2DM, those with fracture had non-significantly higher porosity than those without fracture $(\mathrm{p}=0.159$, Fig. 4 , Table 3$)$. In women without T2DM, those with fracture had higher porosity than those without fracture $(\mathrm{p}<0.001)$. In women with fracture, those with T2DM had non-significantly lower cortical porosity than those without T2DM $(p=0.385)$. In women without fracture, those with T2DM had lower porosity than those without T2DM $(p=0.048)$. All analysis were adjusted for age and BMI.

In stratified analysis of women without T2DM, those with fracture were taller, had lower BMI, higher serum PINP and CTX, lower vitamin D, higher PTH, lower aBMD of the total hip and femoral neck, lower total and cortical vBMD, and thinner cortices of femoral subtrochanteric site than those without fracture (all $p<0.05$, Table 3 ). In stratified analysis of women with T2DM, there were similar trends, although few associations achieved statistical significance.

\section{Discussion}

We report that women with T2DM exhibited lower cortical porosity of the femoral subtrochanteric region than nondiabetic women. This was quantified using low-resolution clinical CT. Women with T2DM had higher total vBMD and cortical vBMD than nondiabetic women. The bone formation marker PINP tended to be lower in those with T2DM than nondiabetic women but did not reach statistical significance. Women with T2DM had higher serum glucose, insulin, and BMI compared to nondiabetic women, and increasing glucose and BMI were associated with lower PINP and CTX. Increasing glucose was associated with reduced cortical porosity, whereas increasing BMI was associated with thicker cortices, thus it 
seems that both increased glucose levels and BMI contributed to preserving the architecture of the cortical bone.

The reasons why patients with T2DM have increased risk of fracture, despite normal BMD are still poorly elucidated. Higher cortical porosity has been invoked as a possible factor increasing fracture propensity [11-13,16]. In a pilot study, 19 postmenopausal women with T2DM had higher cortical porosity at the distal radius than 19 controls (4.3\% vs. 1.9\%) [13]. However, this difference in porosity was reduced after exclusion of two fracture patients with T2DM who had 2-fold greater porosity than the group mean, and the authors suggested that the high porosity in the T2DM group could be attributed to the contribution of the fracture subjects [13]. In another study, 22 African-American women with T2DM exhibited higher cortical porosity of the distal radius than 78 nondiabetic controls (2.9\% vs. $2.3 \%)$ [12]. Postmenopausal women with T2DM of more than 10 years duration, had a non-significant tendency towards higher radial cortical porosity compared to controls (3.0\% vs $2.2 \%$ ) [16], while 11 older women (72-81 years) with diabetes had higher radial porosity than 144 nondiabetic controls (4.3\% vs 3.4\%) [11]. These data suggest a tendency towards increased cortical porosity of the distal radius in women with T2DM in studies with limited number of participants using HR-pQCT. However, the absolute differences in cortical porosity between those with and without T2DM were small, and the cortical thickness was below $1 \mathrm{~mm}$ at the distal radius [11-13,34]. Moreover, HR-pQCT present low values of porosity (1-15\%) because of quantifying only porosity of the compact cortices and only pores over $100 \mu \mathrm{m}$ in diameter, and the threshold based segmentation may misclassify trabecularised cortical bone as trabecular bone $[23,24,26,32]$.

In contrast to previous reports on higher cortical porosity of distal radius using HR-pQCT, we report lower cortical porosity of the proximal femoral shaft using low-resolution CT, in a small 
sample of 22 women with T2DM than in nondiabetic women. The large control group of 421 nondiabetic women added statistical power to the analysis. These results remained unchanged after exclusion of outliers with the highest levels of glucose. Moreover, in a recent study using HR-pQCT for assessment of microarchitecture, the distal radial cortical porosity was lower in 99 women with T2DM than in 316 nondiabetic women $(1.5 \%$ vs $2.0 \%, p=0.001)$ [35]. Thus, women with T2DM have lower porosity when measured at a peripheral site as well as a central site, and by using HR-pQCT as well as low resolution CT imaging technology.

Patsch et al. reported higher cortical porosity of the distal radius and tibia in women with T2DM and fragility fractures compared to those with T2DM without fractures, but a non-significant tendency of lower cortical porosity at most sites when comparing diabetic to nondiabetic women [36]. The higher cortical porosity in women with T2DM with fracture than T2DM without fracture, is similar to prior reports of higher porosity in fracture cases than fracture-free controls $[33,37,38]$. Patsch et al. did not report higher porosity in patients with T2DM than in those without T2DM. In fact, they reported that T2DM patients without fracture had the lowest cortical porosity of all groups, and they had a non-significantly lower porosity than non-diabetic women. In agreement with Patsch et al., we report that women with fracture had higher porosity than those without fracture, while women with T2DM had lower porosity than nondiabetic women. Moreover, cortical porosity is suggested to be increased only in T2DM patients with microvascular complications, however, one limitation in that study was that fracture status was not taken into account [34]. They reported no correlation between bone structure and duration of disease, which is in agreement with our findings of a non-significant association between cortical porosity and diabetes duration. Nevertheless, taking together findings from prior studies and the current study, robust evidence for higher or lower cortical porosity in larger groups of patients with T2DM than in nondiabetic controls, is still lacking. 
We used CT and StrAx1.0 software for quantification of bone architecture [22]. StrAx1.0 is a non-threshold based algorithm, which takes the transitional zone into account and is thus more inclusive than HR-pQCT. This is important because there is a gradual change in attenuation from the outer to the inner part of the bone, and it is a challenge to separate cortical from trabecular bone accurately [24]. By recognizing the transitional zone, we avoid misclassification of trabecularised cortex as trabecular bone $[23,25]$. Moreover, the StrAx 1.0 algorithm does not quantify the number or size of pores within the cortex, but the fraction of void volume within the total cortical bone volume. Thus the partial volume effect is taken into account by including porosity in voxels containing both void volume and bone matrix, regardless of the pore size [23,24]. Studies using threshold-based methods for assessment of cortical porosity present lower values of porosity (between $1 \%$ and $15 \%$ ) because of quantifying only porosity of the compact-appearing cortex and only pores above $100 \mu \mathrm{m}$ in diameter [6,24]. This again may partly explain the differences in the results reported in the current study compared to previous studies of cortical porosity in patients with T2DM.

As high bone turnover is reported to be associated with increased cortical porosity $[18,19]$, it is hard to explain how patients with T2DM can have low bone turnover and increased cortical porosity [11-13,16,36]. Previous reports have been consistent, and reported lower levels of BTM in subjects with T2DM compared to nondiabetic subjects $[6,16,21,39]$. In insulin resistant [40], obese [17], and healthy subjects [15], glucose-loading is reported to exhibit a suppressive effect on BTM. An oral glucose tolerance test suppressed PINP and CTX by two-fourfold, higher fasting serum levels of glucose were associated with lower PINP, and higher levels of insulin were associated with increased bone formation markers [15]. We confirmed that higher glucose levels and higher BMI were associated with lower serum PINP and CTX. 
In the current study, higher glucose was associated with lower cortical porosity, suggesting glucose to be involved in the pathophysiological mechanisms of changes in cortical bone. This association is novel, but weak, and needs to be confirmed in larger studies. We did not measure glycosylated hemoglobin and glucose does not necessarily reflect the long-term hyperglycemia. However, glucose was measured at a standardized setting in fasting morning samples. BMI was not associated with lower cortical porosity, but with thicker cortices. As there is a tight association between obesity, insulin resistance and T2DM development this is not surprising, as thicker tibial cortices and lower tibial cortical porosity is reported in obese women [41]. Generally, obesity has been considered protective against fractures, but studies in the last decade have shown that obese individuals have greater risk of several types of fracture [42]. When adjusted for BMD, increasing BMI is protective for hip fracture only $[43,44]$.

What is the reason for increased fracture propensity in T2DM, if not increased cortical porosity? Although low bone turnover rate, as reflected by low BTM, is associated with reduced risk of fracture, there might be both advantages and disadvantages to low bone turnover. As an example, patients treated over several years with anti-resorptive agents who suffer an atypical femoral fracture, are suggested to have reduced cortical porosity and more mineralized bone matrix at the distal radius [45]. Bone qualities and strength are dependent on many material and structural features, as the collagen cross-linking, mineralization and collagen content $[15,46]$. In patients with T2DM, cross-linking of collagen and secondary mineralization might affect bone qualities negatively, despite of low bone remodeling that might slow loss of bone $[15,47]$. Another possible explanation of deteriorated bone quality in T2DM, is accumulation of advanced glycation end products (AGEs) in the bone matrix because of high glucose levels, giving increased brittleness of collagen, and thereby microdamage $[4,15]$. It is also well 
established that AGEs increase with age and that glycation is associated with altered osteoblast activity [9], and high glucose in vitro might increase apoptosis of osteoblasts $[15,48]$.

The reasons for skeletal fragility in T2DM are not completely understood, and the pathogenesis of the bone fragility in T2DM is likely multifactorial. In addition to insulin resistance, and hyperglycemia with accumulation of AGEs and subsequent impaired bone material properties to brittleness of collagen, other factors may be important. Obesity with accompanying increased visceral fat, and oxidative stress can lead to altered structure and strength of bone, and late stages of diabetes with failure of $\beta$ cell as well as vascular complications. Both obesity and chronic hyperglycemia can lead to changes in the osteoblast receptor signaling [6]. For patients with T2DM, the diabetic complications as neuropathy and retinopathy might cause falls, leading to fracture [6]. However, a large study of 124,655 fracture cases and 373,962 controls reported that diabetes in general carry an increased fracture risk, but diabetic complications did not explain the overall fracture risk [49]. Women with T2DM still exhibited greater risk for fracture after adjustment for impaired vision and fall [50]. In the current study, women with T2DM did not report more falls in the last 12 months than did nondiabetic women. Moreover, duration of T2DM was not associated with higher cortical porosity in the present study, but in fact a non-significant trend of lower cortical porosity.

The strength of this study is that it is based on a general population, with high participation rate, [27], and the diagnosis of T2DM was confirmed based on medical records. The StrAx1.0 software for quantification of bone architecture is validated by confirming strong correlation between measurements using CT and HR-pQCT, and also by rescanning a human hip phantom using the same CT machines as used for the participants of the study with good reproducibility 
[22]. The measurements were obtained from the femoral subtrochanteric region, a central site that consists of a thicker cortex so that cortical architecture could be well quantified.

The limited number of T2DM patients in this study is a limitation, however, the large group of controls adds strength to the statistical analysis. The retrospective case-control design may have introduced selection bias, so it is possible that the strength of the associations is somewhat underestimated due to a "healthy" selection bias. Information from the self-administered questionnaires might be prone to recall bias, resulting in over- or under-reporting. Furthermore, the subtrochanteric site contained little trabecular bone, so the possibility to test its association with diabetes or other factors was limited.

In conclusion, women with type 2 diabetes mellitus had lower cortical porosity of the femoral shaft than nondiabetic women, assessed using low-resolution CT and StrAx1.0 software. Cortical porosity assessed using this software includes porosity not only of the compact cortex, but also the transitional zone, and is thus more inclusive than other methods. Increasing glucose and BMI were associated with reduced bone turnover markers. Reduced bone turnover will lead to reduced intracortical and endocortical remodeling and reduced porosity of thicker cortices. Such changes in bone features would be expected to reduce fracture risk. Increasing cortical porosity is thus unlikely to explain the increased fracture propensity in women with type 2 diabetes. Therefore, other factors as alterations in bone material composition or increased microdamage due to reduced bone turnover are more likely contributors to reduced bone qualities and increased fracture risk in these patients. 


\section{Disclosures}

EFE reported that he has received lecture fees from Amgen, Novartis, Eli Lilly and IDS. All authors state that they have no other conflict of interest.

\section{Acknowledgement}

The North Norwegian Health Authorities funded the study (ID 5645, ID 9167, ID 9168, ID 10295) but had no role in design and conduct of the study; in the collection, analyses, and interpretation of the data; or in the preparation, review, or approval of the manuscript. The Troms $\varnothing$ Study provided access to data. Staff at the Clinical Research Unit at the University Hospital of North Norway (UNN) recruited women, obtained consent and questionnaires, collected blood samples and performed DXA scanning. Staff at the Department of Radiology and Department of Radiation, UNN performed CT scanning of the patients, organized the scanning procedures, and Strax Corp, Melbourne analyzed the CT images.

Authors' roles: Study concept, design, obtained funding and executed the study: MO, RK, ÅB. Statistical analysis: MO, ̊̊B. Drafting manuscript: MO, RK, TTB, IKH, RMJ, EFE, ÅB. Data interpretation and critical revision of the manuscript for important intellectual content, writing of the report and approval of the final version: MO, RK, TTB, IKH, RMJ, EFE, ÅB. ÅB takes responsibility for the integrity of the data analyses. 


\section{References}

[1] E. Hernlund, A. Svedbom, M. Ivergård, J. Compston, C. Cooper, J. Stenmark, et al., Osteoporosis in the European Union: medical management, epidemiology and economic burden, Arch. Osteoporos. 8 (2013) 1-115.

[2] S. Wild, G. Roglic, A. Green, R. Sicree, H. King, Global prevalence of diabetes estimates for the year 2000 and projections for 2030, Diabetes Care 27 (2004) 1047-1053.

[3] A. Svedbom, E. Hernlund, M. Ivergård, J. Compston, C. Cooper, J. Stenmark, et al., Osteoporosis in the European Union: a compendium of country-specific reports, Arch. Osteoporos. 8 (2013) 1-218.

[4] W.D. Leslie, M.R. Rubin, A.V. Schwartz, J.A. Kanis, Type 2 diabetes and bone, J. Bone Miner. Res. 27 (2012) 2231-2237.

[5] P. Vestergaard, Discrepancies in bone mineral density and fracture risk in patients with type 1 and type 2 diabetes - a meta-analysis, Osteoporos. Int. 18 (2007) 427-444.

[6] V.V. Shanbhogue, D.M. Mitchell, C.J. Rosen, M.L. Bouxsein, Type 2 diabetes and the skeleton: new insights into sweet bones, Lancet Diabetes Endocrinol. 4 (2016) 159-173.

[7] M.R. Rubin, J.M. Patsch, Assessment of bone turnover and bone quality in type 2 diabetic bone disease: current concepts and future directions, Bone Res. 4 (2016) 16001.

[8] A.V. Schwartz, E. Vittinghoff, D.C. Bauer, T.A. Hillier, E.S. Strotmeyer, K.E. Ensrud, et al., Association of BMD and FRAX score with risk of fracture in older adults with type 2 diabetes, JAMA. 305 (2011) 2184-2192.

[9] L. Karim, M.L. Bouxsein, Effect of type 2 diabetes-related non-enzymatic glycation on bone biomechanical properties, Bone 82 (2016) 21-27.

[10] U. Heilmeier, D.R. Carpenter, J.M. Patsch, R. Harnish, G.B. Joseph, A.J. Burghardt, et al., Volumetric femoral BMD, bone geometry, and serum sclerostin levels differ between type 2 diabetic postmenopausal women with and without fragility fractures, Osteoporos. Int. 26 (2015) 1283-1293.

[11] J. Paccou, K.A. Ward, K.A Jameson, E.M. Dennison, C. Cooper, M.H. Edwards, Bone Microarchitecture in Men and Women with Diabetes: The Importance of Cortical Porosity, Calcif. Tissue Int. 98 (2016) 465-473.

[12] E.W. Yu, M.S. Putman, N. Derrico, G. Abrishamanian-Garcia, J.S. Finkelstein, M.L. Bouxsein, Defects in cortical microarchitecture among African-American women with type 2 diabetes, Osteoporos. Int. 26 (2015) 673-679.

[13] A.J. Burghardt, A.S. Issever, A.V. Schwartz, K.A. Davis, U. Masharani, S. Majumdar, T.M. Link, High-resolution peripheral quantitative computed tomographic imaging of cortical and trabecular bone microarchitecture in patients with type 2 diabetes mellitus, $\mathrm{J}$ Clin. Endocrinol. Metab. 95 (2010) 5045-5055.

[14] P. Gerdhem, A. Isaksson, K. Åkesson, K.J. Obrant, Increased bone density and decreased bone turnover, but no evident alteration of fracture susceptibility in elderly women with diabetes mellitus, Osteoporos. Int. 16 (2005) 1506-1512.

[15] I. Levinger, E. Seeman, G. Jerums, G.K. McConell, M.S. Rybchyn, S. Cassar, et al., Glucose-loading reduces bone remodeling in women and osteoblast function in vitro, Physiol. Rep. 4 (2016) e12700.

[16] J.N. Farr, M.T. Drake, S. Amin, L.J. Melton, L.K. McCready, S. Khosla, In vivo assessment of bone quality in postmenopausal women with type 2 diabetes, J. Bone Miner. Res. 29 (2014) 787-795.

[17] P. Iglesias, F. Arrieta, M. Pinera, J. Botella-Carretero, J. Balsa, I. Zamarron, et al., Serum concentrations of osteocalcin, procollagen type $1 \mathrm{~N}$-terminal propeptide and betaCrossLaps in obese subjects with varying degrees of glucose tolerance, Clinical Endocrinology 75 (2011) 184-188. 
[18] R. Shigdel, M. Osima, L.A. Ahmed, R.M. Joakimsen, E.F. Eriksen, R. Zebaze, A. Bjørnerem, Bone turnover markers are associated with higher cortical porosity, thinner cortices, and larger size of the proximal femur and non-vertebral fractures, Bone 81 (2015) 1-6.

[19] A. Bjørnerem, A. Ghasem-Zadeh, M. Bui, X. Wang, C. Rantzau, T.V. Nguyen, et al., Remodeling markers are associated with larger intracortical surface area but smaller trabecular surface area: a twin study, Bone 49 (2011) 1125-1130.

[20] J. Starup-Linde, S. Eriksen, S. Lykkeboe, A. Handberg, P. Vestergaard, Biochemical markers of bone turnover in diabetes patients - a meta-analysis, and a methodological study on the effects of glucose on bone markers, Osteoporos. Int. 25 (2014) 1697-1708.

[21] N. Napoli, M. Chandran, D.D. Pierroz, B. Abrahamsen, A.V. Schwartz, S.L. Ferrari, Mechanisms of diabetes mellitus-induced bone fragility, Nat. Rev. Endocrinol. 2016 [Epub ahead of print] doi:10.1038/nrendo.2016.153

[22] L.A. Ahmed, R. Shigdel, R.M. Joakimsen, O.P. Eldevik, E.F. Eriksen, A. Ghasem-Zadeh, et al., Measurement of cortical porosity of the proximal femur improves identification of women with nonvertebral fragility fractures, Osteoporos. Int. 26 (2015) 2137-2146.

[23] R. Zebaze, A. Ghasem-Zadeh, A. Bohte, S. Iuliano-Burns, M. Mirams, R.I. Price, et al., Intracortical remodelling and porosity in the distal radius and post-mortem femurs of women: a cross-sectional study, Lancet 375 (2010) 1729-1736.

[24] R. Zebaze, A. Ghasem-Zadeh, A. Mbala, E. Seeman, A new method of segmentation of compact-appearing, transitional and trabecular compartments and quantification of cortical porosity from high resolution peripheral quantitative computed tomographic images, Bone 54 (2013) 8-20.

[25] R. Zebaze, E. Seeman, Cortical bone: a challenging geography, J. Bone Miner. Res. 30 (2015) 24-29.

[26] А. Bjørnerem, The clinical contribution of cortical porosity to fragility fractures, BoneKEy Rep. 5 (2016) 846.

[27] B.K. Jacobsen, A.E. Eggen, E.B. Mathiesen, T. Wilsgaard, I. Njølstad, Cohort profile: the Troms $\varnothing$ study, Int. J. Epidemiol. 41 (2012) 961-967.

[28] ̊̊. Bjørnerem, L.A. Ahmed, L. Jørgensen, J. Størmer, R.M. Joakimsen. Breastfeeding protects against hip fracture in postmenopausal women: The Troms $\varnothing$ study, J. Bone Miner. Res. 26 (2011) 2843-2850.

[29] R. Shigdel, M. Osima, M. Lukic, L.A. Ahmed, R.M. Joakimsen, E.F. Eriksen, et al., Determinants of Transitional Zone Area and Porosity of the Proximal Femur Quantified In Vivo in Postmenopausal Women, J. Bone Miner. Res. 31 (2015) 758-766.

[30] D. Matthews, J. Hosker, A. Rudenski, B. Naylor, D. Treacher, R. Turner, Homeostasis model assessment: insulin resistance and $\beta$-cell function from fasting plasma glucose and insulin concentrations in man, Diabetologia 28 (1985) 412-419.

[31] R. Shigdel, Cortical porosity as a target for fracture prevention: The Troms $\varnothing$ Study, Thesis/Dissertation, 2016 ISBN 9788275894968.

[32] R. Zebaze, C. Libanati, M.R. McClung, J.R. Zanchetta, D.L. Kendler, A. Høiseth, et al., Denosumab reduces cortical porosity of the proximal femoral shaft in postmenopausal women with osteoporosis, J. Bone Miner. Res. 31 (2016) 1827-1834.

[33] Å. Bjørnerem, Q.M. Bui, A. Ghasem-Zadeh, J.L. Hopper, R. Zebaze, E. Seeman, Fracture risk and height: an association partly accounted for by cortical porosity of relatively thinner cortices, J. Bone Miner. Res. 28 (2013) 2017-2026.

[34] V.V. Shanbhogue, S. Hansen, M. Frost, N.R. Jørgensen, A.P. Hermann, J.E. Henriksen, et al., Compromised cortical bone compartment in type 2 diabetes mellitus patients with microvascular disease, Eur. J. Endocrinol. 174 (2016) 115-124. 
[35] A.G. Nilsson, D. Sundh, M. Nilsson, R. Rudäng, A. Darelid, M. Zoulakis, et al., Type 2 Diabetes Mellitus Is Associated with Better Bone Microarchitecture but Lower Bone Material Strength and Poorer Physical Function in Elderly Women - a Population-Based Study, J. Bone Miner. Res. 2016 [Epub ahead of print] doi: 10.1002/jbmr.3057

[36] J.M. Patsch, A.J. Burghardt, S.P. Yap, T. Baum, A.V. Schwartz, G.B. Joseph, et al., Increased cortical porosity in type 2 diabetic postmenopausal women with fragility fractures, J. Bone Miner. Res. 28 (2013) 313-324.

[37] K.F. Bell, N. Loveridge, J. Power, N. Garrahan, B.F. Meggitt, J. Reeve, Regional differences in cortical porosity in the fractured femoral neck, Bone 24 (1999) 57-64.

[38] Y. Bala, R. Zebaze, A. Ghasem-Zadeh, E.J. Atkinson, S. Iuliano, J.M. Peterson, et al., Cortical Porosity Identifies Women with Osteopenia at Increased Risk for Forearm Fractures, J. Bone Miner. Res. 29 (2014) 1356-1362.

[39] A. Shu, M.T. Yin, E. Stein, S. Cremers, E. Dworakowski, R. Ives, M.R. Rubin, Bone structure and turnover in type 2 diabetes mellitus, Osteoporos. Int. 23 (2012) 635-641.

[40] V. Schwetz, E. Lerchbaum, N. Schweighofer, N. Hacker, O. Trummer, O. Borel, et al., Osteocalcin levels on oral glucose load in women being investigated for polycystic ovary syndrome, Endocr. Pract. 20 (2013) 5-14.

[41] A.L. Evans, M.A. Paggiosi, R. Eastell, J.S. Walsh, Bone density, microstructure and strength in obese and normal weight men and women in younger and older adulthood, J. Bone Miner. Res. 30 (2015) 920-928.

[42] J.E. Compston, N.B. Watts, R. Chapurlat, C. Cooper, S. Boonen, S. Greenspan, et al., Obesity is not protective against fracture in postmenopausal women: GLOW, Am. J. Med. 124 (2011) 1043-1050.

[43] H. Johansson, J.A. Kanis, A. Odén, E. McCloskey, R.D. Chapurlat, C. Christiansen, et al., A meta-analysis of the association of fracture risk and body mass index in women, $\mathrm{J}$. Bone Miner. Res. 29 (2014) 223-233.

[44] D. Prieto-Alhambra, M.O. Premaor, F. Fina Avilés, E. Hermosilla, D. Martinez-Laguna, C. Carbonell-Abella, et al., The association between fracture and obesity is sitedependent: a population-based study in postmenopausal women, J. Bone Miner. Res. 27 (2012) 294-300.

[45] C. Chiang, E. Seeman, A. Ghasem-Zadeh, S. Juliano, P. Ebeling, H. Nguyen, et al., Bone's material composition and microstructure are compromised in women sustaining atypical femoral fractures during antiresorptive therapy, J. Bone Miner. Res. (Suppl. 1) (2015) 30.

[46] M.L. Bouxsein, Bone quality: where do we go from here? Osteoporos. Int. 14 (2003) 118127.

[47] D. Vashishth, The role of the collagen matrix in skeletal fragility, Curr. Osteoporos. Rep. 5 (2007) 62-66.

[48] M. Inaba, M. Terada, H. Koyama, O. Yoshida, E. Ishimura, T. Kawagishi, et al., Influence of high glucose on 1, 25-dihydroxyvitamin D3-induced effect on human osteoblast-like MG-63 cells, J. Bone Miner. Res. 10 (1995) 1050-1056.

[49] P. Vestergaard, L. Rejnmark, L. Mosekilde, Diabetes and its complications and their relationship with risk of fractures in type 1 and 2 diabetes, Calcif. Tissue Int. 84 (2009) 45-55.

[50] D.E. Bonds, J.C. Larson, A.V. Schwartz, E.S. Strotmeyer, J. Robbins, B.L. Rodriguez, et al., Risk of fracture in women with type 2 diabetes: the Women's Health Initiative Observational Study, J. Clin. Endocrinol. Metab. 91 (2006) 3404-3410. 